\title{
STABLE COMPLEX MANIFOLDS ${ }^{1}$
}

\author{
BY ALLAN BRENDER ${ }^{2}$
}

Communicated by E. Spanier, July 14, 1966

1. T. Van de Ven [3] has recently shown that there exist real 4dimensional manifolds which admit almost complex structures but admit no complex structures, e.g. $S^{1} \times S^{3} \# S^{1} \times S^{3} \# C P(2)$. The purpose of this note is to show that this is an unstable phenomenon.

Let $M^{n}$ be a $C^{\infty} n$-dimensional real manifold without boundary and let $\tau_{M}$ be its tangent bundle. $R^{k}$ is real Euclidean $k$-space and $C^{k}$ is complex $k$-space.

Definition 1. $M^{n}$ admits a stable complex structure if $M^{n} \times R^{k}$ can be given the structure of a complex analytic manifold for some $k \geqq 0$, $n \equiv k(\bmod 2)$.

Let $\xi^{m}$ be an $m$-plane bundle over $M^{n}$.

Definition 2. A stable complex structure for $\xi^{m}$ is a reduction of the group of $\xi^{m} \oplus \epsilon^{k}$ to $U((m+k) / 2)$ for some $k \geqq 0, m \equiv k(\bmod 2)$.

Definition 3. A stable almost complex structure for $M^{n}$ is a stable complex structure for $\tau_{M}$.

Proposition. $M^{n}$ admits a stable complex structure if and only if it admits a stable almost complex structure.

2. It is clear that a stable complex structure carries with it a stable almost complex structure. We show the converse is true.

We can assume $M^{n}$ is a real analytic manifold. There exists a complex $n$-dimensional manifold $N_{c}^{n}$ (of real dimension $2 n$ ) and a real analytic embedding $i: M^{n} \subset N_{C}^{n}$ [4]. Regarding $N$ as a real manifold, it is easy to see from the construction of [4] that $\nu(i)$, the normal bundle of the embedding, is equivalent to $\tau_{M}$; i.e., $\tau_{N} \mid M \approx \tau_{M} \oplus \tau_{M}$. Let $U \subset N$ be a tubular neighborhood of $M$ in $N$ and let $r: U \rightarrow M$ be the bundle projection ( $U$ is identified with the total space of $\nu(i)$ ). One can construct an open neighborhood, $V$, of $M$ in $U(M \subset V \subset U \subset N)$ which is a domain of holomorphy [2]. Let $r: V \rightarrow M$ be the restriction of $r: U \rightarrow M$.

Let $\nu^{2 n+1}$ be the stable normal bundle to $M^{n}$. $\tau_{M} \oplus \nu^{2 n+1} \approx \epsilon^{3 n+1}$. $\tau_{M}$ admits a stable complex structure if and only if $\nu$ does. Assume now that there is a bundle $\sigma$ over $M$ with fiber $C^{m}$ and group $U(m)$

${ }^{1}$ Research supported in part by the National Science Foundation. These results are contained in the author's doctoral dissertation submitted to the University of California, Berkeley.

2 The author wishes to thank Professors Phillip Griffiths and Morris Hirsch for several very helpful conversations. 
and an $R^{2 m}$-bundle equivalence $f: \sigma \rightarrow \nu \oplus \epsilon^{k}, 2 n+k+1=2 m$. We have $r^{*} \sigma$ over $V$. By Satz II of [1], we can assume $r^{*} \sigma$ is a holomorphic vector bundle over $V$. Let $\Pi: E \rightarrow V$ be the bundle projection. Considering $E$ as a real analytic manifold we have

$$
\begin{aligned}
\tau_{E} \mid M & \approx \Pi^{*} \tau_{V}\left|M \oplus \Pi^{*} r^{*} \sigma\right| M \\
& \approx \tau_{V}\left|M \oplus r^{*} \sigma\right| M \\
& \approx \tau_{N} \mid M \oplus \sigma \\
& \approx \tau_{M} \oplus \tau_{M} \oplus \nu \oplus \epsilon^{k} \\
& \approx \tau_{M} \oplus \epsilon^{3 n+k+1} .
\end{aligned}
$$

Therefore the embedding $M \subset V \subset E$ has a trivial normal bundle. $M^{n} \times R^{3 n+k+1}$ can now be regarded as an open subset of $E$ and so it inherits the structure of a complex manifold from the complex structure on E. Q.E.D.

\section{REFERENCES}

1. H. Grauert, Analytische Faserungen über hollomorph-vollständigen Räumen, Math. Ann. 135 (1958), 263-275.

2. . - On Levi's problem and the imbedding of real-analytic manifolds, Math. Ann. 68 (1958), 460-472.

3. T. Van de Ven (to appear).

4. H. Whitney and F. Bruhat, Quelques propriêtés fondamentales des ensembles analytiques-réels, Comment. Math. Helv. 33 (1959), 132-160.

University of California, Berkeley 